\title{
Educación ambiental y medios de comunicación: revisión de la literatura y propuestas de intervención
}

\author{
F. Javier Perales Palacios \\ Departamento de Didáctica de las Ciencias Experimentales. Universidad de Granada. \\ fperales@ugr.es \\ ORCID: https://orcid.org/0000-0002-6112-2779
}

[Recibido: 16 Abril 2020. Revisado: 3 Julio 2020. Aceptado: 11 Julio 2020]

\begin{abstract}
Resumen: Hoy en día gran parte de la información sobre temas ambientales que reciben los ciudadanos lo hacen a través de los medios de comunicación (MC) y, en especial, de los distintos canales que ofrece Internet. En este artículo queremos responder a dos cuestiones: (1) ¿qué nos dice la literatura respecto a las características de dicha información y las propuestas educativas que pudieran derivarse?; (2) ¿cómo utilizar los MC como instrumento para promover los fines propios de la Educación Ambiental (EA)? La revisión de la literatura permite establecer una serie de características positivas y negativas de la información ambiental en los MC. Se concluye con una propuesta de integración de los MC como recurso para la ambientalización del currículo educativo.
\end{abstract}

Palabras clave: Educación ambiental; medios de comunicación; ambientalización curricular.

Environmental Education and the media: Review of the literature and proposals for intervention

\begin{abstract}
Today, much of the information on environmental issues received by citizens is provided through the media and, in particular, through the various channels offered by the Internet. In this article we want to answer two questions: (1) what does the literature tell us about the characteristics of this information and the educational proposals that could be derived? ; (2) how can the media be used as a tool to promote the proper purposes of Environmental Education? The literature review allows to establish a number of positive and negative characteristics of environmental information in the media. It concludes with a proposal for the integration of the media as a resource for the environment of the educational curriculum.
\end{abstract}

Keywords: Environmental education; media; environment of the educational curriculum.

Para citar este artículo: Perales, F. J. (2020). Educación Ambiental y medios de comunicación: revisión de la literatura y propuestas de intervención. Revista de Educación Ambiental y Sostenibilidad 2(2), 2102. doi: 10.25267/Rev_educ_ambient_sostenibilidad.2020.v2.i2.2102

\section{Introducción}

Desde que la Unión Internacional de Conservación de la Naturaleza (UICN, 1970) definiera la EA como:

El proceso que consiste en reconocer valores y aclarar conceptos con objeto de fomentar las aptitudes $y$ actitudes necesarias para comprender y apreciar las interrelaciones entre el hombre, su cultura y su medio biofísico. Entraría también la práctica en la toma de decisiones y en la propia elaboración de un código de comportamiento con respecto a las cuestiones relacionadas con la calidad del Medio Ambiente 
han transcurrido casi cinco décadas, pero no ha perdido ninguna vigencia. Nadie discute que las señas de identidad de la EA estriban en su vertiente actitudinal, pero a partir, como señala la definición anterior, de "aclarar conceptos, fomentar aptitudes y comprender las interrelaciones". Es decir, una EA relevante tiene que incluir como condición necesaria, aunque no suficiente, una información significativa sobre los problemas ambientales y sus implicaciones sociales, dado su carácter predictor de la preocupación, actitudes y responsabilidad ambiental (Teksoz, Sahin y TekkayaOztekin, 2012). Pero, ¿cuáles son las fuentes de esa información que calificamos como imprescindibles?

Dada la progresiva concentración urbana que experimenta la humanidad y el desapego constante del medio natural, al margen de las diferencias entre países en su incorporación de la EA a los currículos oficiales, del papel de las familias y de las relaciones sociales, la mayor parte de esa información es la que proporcionan los MC de masas. De aquí surgen las siguientes preguntas de investigación:

(1) ¿Qué características posee esa información?

(2) ¿Satisface los requerimientos para cumplir con una labor alfabetizadora de la ciudadanía?

(3) ¿Podríamos integrar los MC en los currículos oficiales de la educación básica?

(4) ¿Cómo llevarlo a cabo?

Este estudio representa un aporte más en una línea de investigación que iniciamos en el año 1999 (Perales y García, 1999), donde proponíamos la integración sistemática en el currículo escolar de los MC mediante algunos ejemplos. Posteriormente (Perales, 2010) profundizamos en esta temática, indagando en las características de la información ambiental que transmiten los MC, en general, y la prensa y la televisión, en particular, así como analizamos las dificultades a las que se enfrenta y planteamos cómo debería ser. En el presente artículo nos proponemos ampliar la fundamentación teórica sobre el papel de los MC en la EA y concretar propuestas de integración curricular.

\section{Marco teórico}

Los MC constituyen actualmente lo que se ha llamado el cuarto poder. Desde la aparición de la radio, el cine y posteriormente la Tv, estos MC han adquirido la denominación de masas por la cantidad y heterogeneidad de la audiencia, habiendo superado ampliamente a los periódicos y los libros. Actualmente Internet prácticamente ha fagocitado a todos ellos mediante aplicaciones y canales como los libros electrónicos, periódicos digitales, pódcast, Youtube, Netflix... Y a ello no podría ser ajena la información ambiental y, por ende, la EA.

Paraskeva-Hadjichambi et al. (2020) enumeran diferentes formas de adquirir información ambiental (tabla 1). Entre ellas, los medios audiovisuales, los impresos y los electrónicos entrarían en la categoría de los MC.

Pero no solo los MC han sustituido al papel que debería jugar la escuela, sino que en muchas ocasiones dichos papeles son antagónicos. De hecho, como afirma Quintanar (2013), "para la EA, sobre todo formal, es que lo que se diga y practique en la escuela, 
a nivel formal, se enfrenta directamente a lo que propaguen los MC, en una lucha muy desigual" (p. 8).

Tabla 1. Lugares de libre elección para aprender sobre el entorno. Fuente: adaptada de ParaskevaHadjichambi et al. (2020, p. 215)

\begin{tabular}{ll} 
Grupo genérico & Ejemplos de lugares \\
Museos & Museos de historia natural, centros / museos de ciencia, \\
& zoológicos, acuarios, jardines botánicos, arboretos, centros de \\
& naturaleza \\
& Programas estructurados a veces vinculados al plan de estudios \\
fermal, p. ej., granjas-escuela & Locales, regionales, nacionales \\
Parques & Excursiones de observación de ballenas y marinas, viajes de \\
Sitios de ecoturismo & encuentro con safaris y vida silvestre, refugios en áreas \\
& naturales, expediciones de ciencia ciudadana \\
Medios audiovisuales & Programas de TV, películas y videos, radio \\
Medios impresos & Periódicos, revistas y publicaciones periódicas \\
Medios electrónicos & Internet, redes sociales (Facebook y Twitter), sitios web de \\
& petición (Change.org) \\
Organizaciones ecologistas & WWF, Greenpeace, SEO \\
Clubes y movimientos & Scout \\
Celebración días internacionales & Día de limpieza del mar \\
Entorno familiar & Conversaciones (familia y amigos), vecindario y parques \\
& infantiles \\
\hline
\end{tabular}

Podemos afirmar que los MC son los instrumentos de la comunicación ambiental, un paradigma relativamente nuevo ya que sus precedentes se remontan a la década de los 70 del pasado siglo y han registrado un incremento constante desde entonces hasta nuestros días (Román-Núñez y Cuesta, 2016).

En la figura 1 se muestra el marco conceptual en que se desenvuelve el presente estudio de revisión, al que añadiremos Internet dada su pujanza actual como MC de masas.

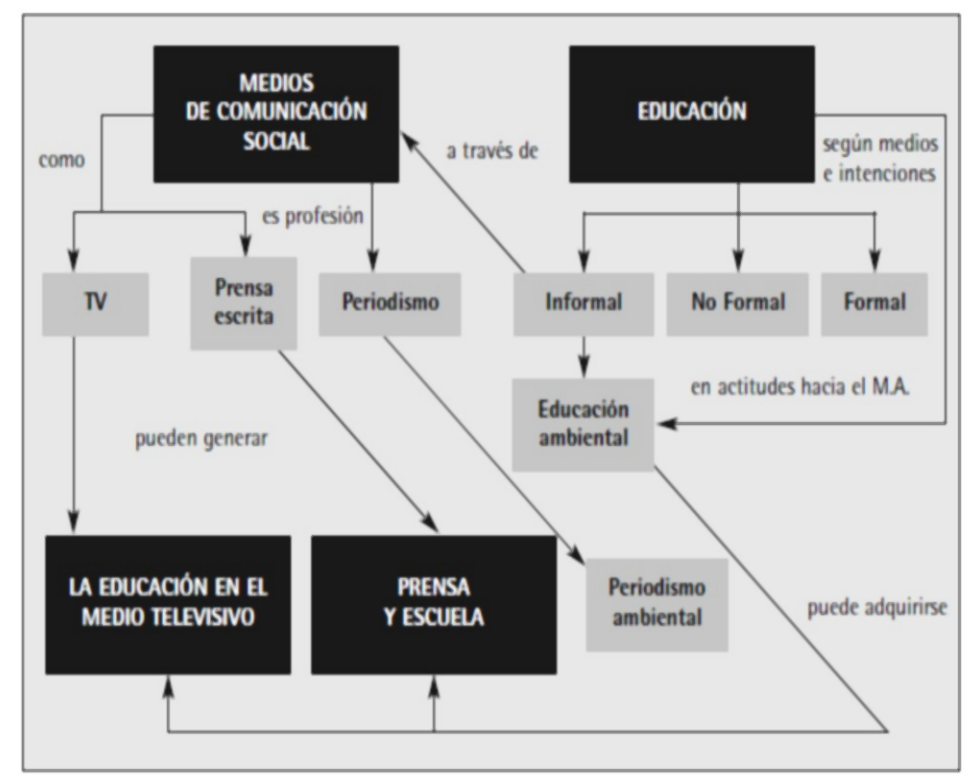

Figura 1. Marco conceptual representativo de la relación entre los MC y la EA. Fuente: Perales (2010, p. 24). 
En la tabla 2 mostramos una primera selección de referencias bibliográficas relativas a la incidencia de los MC en la EA mediante una búsqueda en Google Académico con las palabras clave EA y MC en los títulos de los artículos y en sus resúmenes.

Tabla 2. El papel de los MC en la EA.

\begin{tabular}{|c|c|}
\hline Referencia bibliográfica & Efectos \\
\hline & Positivos \\
\hline Blum, 1987 & Principal fuente de información ambiental \\
\hline Engels y Jacobson, 2007; Teksoz, Boone, Tuzun- & Relevante y creciente influencia en la \\
\hline Oztekin, 2014 & alfabetización ambiental de la ciudadanía \\
\hline Taddicken, 2013 & Actitud ambiental \\
\hline Wakefield y Elliott, 2003 & Percepción del riesgo \\
\hline Takahashi, Tandoc, Duan y Van Witsen, 2017 & Ciudadanía ambiental \\
\hline Weingart, Engelsy Pansegrau, 2000 & Interés público y compromiso político \\
\hline \multirow[t]{2}{*}{ Östman, 2014} & $\begin{array}{l}\text { Comportamientos ambientales y socialización } \\
\text { política de los jóvenes }\end{array}$ \\
\hline & Negativos \\
\hline Barney, Mintzes y Yen, 2004 & $\begin{array}{l}\text { No proporcionan información que sea de valor } \\
\text { directo en la conservación y protección de } \\
\text { grandes grupos de la megafauna carismática }\end{array}$ \\
\hline \multirow[t]{2}{*}{ Östman y Parker, 1987b } & $\begin{array}{l}\text { Valoración negativa de los medios en cuatro } \\
\text { criterios: falta de equilibrio, orientación política } \\
\text { sesgada, sensacionalismo y tendencia a } \\
\text { seleccionar para maximizar la audiencia }\end{array}$ \\
\hline & Ambivalentes \\
\hline Östman y Parker, 1987a & $\begin{array}{l}\text { Influencia positiva sobre el conocimiento, los } \\
\text { intereses y el comportamiento ambiental de los } \\
\text { periódicos, frente una negativa de la TV }\end{array}$ \\
\hline Östman y Parker, 1987b & $\begin{array}{l}\text { Diferencias en cuanto a credibilidad y uso de } \\
\text { ambos medios en función del nivel educativo de } \\
\text { los encuestados }\end{array}$ \\
\hline
\end{tabular}

Lee (2011), basándose en una revisión de la literatura, atribuye la influencia de los MC a tres posibles factores: (1) establecen la agenda ambiental como temas importantes para que la audiencia piense; (2) sugieren a la audiencia los atributos específicos de los temas ambientales importantes a los que prestar atención; o (3) actúan como una fuente de norma social, sugiriendo qué valores y comportamientos son aceptados por otros y cuáles no.

A partir de las anteriores evidencias, otros autores reclaman que los responsables políticos podrían aprovechar esa situación para planificar adecuadamente una EA integral de las masas a través de estos medios, complementaria a la educación formal (Said, Ahmadun, Paim y Masud, 2003).

Una perspectiva más concreta intenta mostrar cómo los $\mathrm{MC}$ presentan algunos problemas ambientales, destacando entre ellos el cambio climático (CC). Así, por ejemplo, Bámaca-López (2016, pp. 29-35) muestra la evolución de la presencia de aquél en los MC y León (2014) aborda desde una perspectiva compleja la relación entre el CC y los MC, insertándola en el contexto más amplio de la comunicación.

Vamos a centrarnos ahora en revisar la especificidad de algunos MC, tales como la prensa (escrita y digital), el formato audiovisual (TV y documentales) y otros. 


\section{Prensa}

Actualmente en su formato electrónico ha ampliado su audiencia gracias a las posibilidades de acceso desde los dispositivos móviles y fijos. La investigación sobre aquella se ha centrado especialmente en el análisis de noticias de problemas ambientales, tanto en el tiempo como confrontando medios informativos. Así, por ejemplo, Quesada, Blanco y Teruel (2015) se ocupan de comparar, mediante un análisis de contenido, el tratamiento del CC en cuatro periódicos pertenecientes a otros cuatro países europeos. En el caso de periódicos mexicanos, Ramos, Molina, Hernández y Sánchez (2011) analizaron cuatro periódicos locales considerando variables como ubicación espacial de las notas, géneros periodísticos, niveles discursivos, contenido temático, componentes visuales y tipo de argumentación, y encontraron algunas tendencias comunes en la línea editorial de cada uno de ellos. Concluyeron que la atención a los temas ambientales es marginal, descontextualizada, ligada a desastres naturales o con agendas locales/estatales. Por su parte, León y Ruiz (2012) analizaron el tratamiento recibido por la sequía en la prensa española en un periodo de cincuenta años y detectaron una progresión en la complejidad de la información y en el de las campañas de concienciación.

Como respuestas innovadoras, destaca el crecimiento de secciones de la prensa especializada en temas ambientales (por ejemplo, Efe Verde ${ }^{1}$ ).

\section{Televisión, cine y documentales}

Aunque el formato audiovisual cada vez invade más MC, en este apartado incluiremos una muestra de la investigación que se viene realizando en torno a la programación televisiva, cine y documentales, aunque puedan también estar soportados por Internet.

Montero (2008) analizó desde una perspectiva global la información ambiental que ofrece la TV. Destaca, en primer lugar, la degradación progresiva que ha experimentado y, entre otros, los siguientes aspectos: su escasa presencia en el global de la programación, el desequilibrio entre la demanda ciudadana y la oferta televisiva, la brevedad de tiempo dedicado a las noticias, su apuesta por el entretenimiento y el índice de audiencia, su oscilación entre la trivialización y el catastrofismo, la preponderancia de contextos lejanos o su preferencia por lo estético (caso de los documentales). Esto, recalca el autor, resulta especialmente grave en las cadenas públicas.

Respecto al volumen de la información ambiental presente en la TV, es de destacar su escasa presencia en las cadenas españolas, ya sea de forma absoluta o considerada dentro de la información científica (Francescutti, 2010).

Fortner y Lyon (1985) analizaron el efecto de la visión de un documental de Cousteau sobre el cambio en el conocimiento y actitud de los televidentes en comparación con el de otras personas que no lo vieron. Los resultados fueron positivos en el grupo experimental pero estos efectos se atenuaron en un periodo de dos semanas.

Brothers, Fortner y Mayer (1991) estudiaron el impacto de la TV en el conocimiento ambiental y Bonnett y William (1998) midieron el impacto de un programa de noticias de TV en la educación de adultos, respondiendo estos posteriormente a

\footnotetext{
${ }^{1}$ https://www.scoop.it/topic/efeverde
} 
preguntas de conocimiento seleccionadas con segmentos explicativos de vídeo, evidenciándose un aumento significativo en los niveles de conocimiento.

Cumba (2020) analizó la programación de un canal de TV ecuatoriano y encontró que presta más atención a los problemas de contaminación que a su prevención y concienciación de la ciudadanía, es decir, a lo que podría considerarse que corresponde al ámbito de la EA.

Bayma (2013) abordó un análisis de contenido comparativo de dos documentales de amplia difusión y con diferente sentido ideológico respecto al estado ambiental de la Tierra. Utiliza para ello tanto su eficacia comunicativa como las críticas recibidas desde distintos ámbitos científicos. Ello viene a mostrar cómo problemas globales como el CC son susceptibles de ser interpretados desde diferentes puntos de vista, tanto racionales como emocionales.

Por su parte, Sancho, Vilches y Gil (2010) revisaron una muestra de documentales científicos en función de la atención que prestaban a unos indicadores previos sobre problemáticas ambientales. Para los autores los resultados muestran que los analizados no proporcionan, en general, una visión global de la situación del mundo.

El impacto de películas de carácter ambiental responde a un patrón similar al de los documentales. Así, Lowe, Brown, Dessai, de França, Haynes y Vincent (2006) analizaron los efectos del visionado de una película sobre la transformación abrupta y catastrófica del clima de la Tierra. Encontraron que ver la película, al menos a corto plazo, cambió las actitudes de las personas, manifestándose más preocupados por el CC y otros riesgos ambientales. Sin embargo, si bien la película aumentó la ansiedad sobre los riesgos ambientales, los espectadores experimentaron dificultades para distinguir los hechos científicos de la ciencia-ficción dramatizada y se redujo su creencia en la probabilidad de eventos extremos como resultado del CC. Tras el visionado de la película, muchos espectadores expresaron una fuerte motivación para actuar sobre el CC pero carecían de información sobre qué medidas podían tomar para mitigarlo.

\section{Otros medios}

Las TIC, en general, ofrecen un amplísimo marco donde albergar toda clase de información ambiental: web, blogs, repositorios, artículos, libros... y a su vez constituyen un soporte de las redes sociales, Twiter, Facebook, Instagram, Whatsapp... Ello ha promovido la generación de nuevas herramientas de evaluación de dicha información.

En un artículo sobre el uso de las TIC por parte de una muestra de educadores ambientales (Ojeda, Gutiérrez-Pérez y Perales, 2011), los resultados evidenciaron una alta valoración de los educadores hacia las TIC, aunque existía un bajo nivel de preparación y se enfrentaban a dificultades técnicas, organizativas, económicas o, incluso, de convicción sobre su utilidad en sustitución del contacto con el medio natural, lo que hacía que el uso de las TIC no fuera lo frecuente que sería deseable ni se aprovecharan sus múltiples posibilidades.

Ojeda, Perales y Gutiérrez-Pérez (2012) llevaron a cabo un análisis empírico de las webs y blogs de temáticas ambientales de mayor impacto mediático en contextos de habla hispana, proponiendo criterios de evaluación basados en su potencial de interactividad con los usuarios, dinamismo, relevancia de contenidos y visibilidad en 
la red. Los resultados apuntan a que las páginas web de EA son poco relevantes y prestan muy poca atención a los aspectos pedagógicos y educativos, focalizándose especialmente en temas puramente ambientales, careciendo de originalidad en forma, contenidos y planteamiento, ya que no consideran las características especiales del aprendizaje mediado por ordenador ni las posibilidades de la Web 2.0, ni tampoco invitan a la participación activa ni creación de redes. Más recientemente (Pérez-Nieto y Perales, 2018) realizaron un estudio analítico de las características informativas del Cambio Climático (CC) en prensa digital y Youtube, con especial énfasis en los errores que incluían y comparándolos con los de la ciudadanía.

En cuanto a las redes sociales, Osorno (2013) reclama su potencialidad como elemento educativo en el aprendizaje colaborativo, cooperativo y en el aprendizaje significativo. Esta autora abordó un estudio con Facebook que, a su juicio, permite potenciar la EA a través de: "crear grupos bajo temáticas ambientales, establecer foros a partir de diversos recursos, publicar y compartir información sobre temas medio ambientales y crear campañas con objetivos conservacionistas" (p. 57). En este mismo contexto, Segerberg y Bennett (2011) analizaron Twiter como soporte de las protestas ecologistas utilizando como referencia dos hashtags y la información que de ellos podría extraerse. Por último, Jiménez-Liso, Perales y Lara-Vargas (2019) analizaron la repercusión de una catástrofe ambiental recurriendo a una comparativa entre las noticias de prensa y el contenido de Twiter. Los resultados mostraron una evolución de la información desde el ámbito periodístico al de los tweets protagonizados preferentemente por los partidos políticos.

\section{¿Qué características posee esa información?}

Si recuperamos la primera cuestión que nos planteábamos en este artículo, Bonnett y Williams (1998) indican que los MC son muy selectivos a la hora de presentar la información ambiental, prevaleciendo visiones sesgadas de los problemas ambientales que impiden que sean utilizados como un adecuado medio educativo. Aunque en los párrafos anteriores ya se han deslizado algunas valoraciones sobre la naturaleza de la información ambiental de los MC, vamos a centrarnos ahora en estudios más específicos.

Román-Núñez y Cuesta (2016) califican a los MC como pasivos, esto es, se alejan de la búsqueda de explicaciones, con un sentido crítico y aportando soluciones catastrofistas, simplistas y mostrando su antagonismo con la humanidad. A su vez, Bell (1994) identificó errores significativos en la información sobre CC en las noticias durante un periodo de seis meses. Por otro lado, Thompson-Saud, Gelcich y Barraza (2018) encontraron que la información sobre conservación marina en periódicos y TV poseía un sesgo hacia intereses comerciales y económicos. Dotson, Jacobson, Kaid y Carlton (2012) detectaron igualmente importantes diferencias en la cantidad, extensión y formato de noticias ambientales en dos periódicos de diferente ideología política. Weingart, Engels y Pansegrau (2000) también hallaron diferencias entre los discursos políticos, científicos y de los MC sobre el CC, lo que califican como los riesgos de la comunicación. Ignoraron las incertidumbres y las transformaron en una secuencia de eventos que conducen a una catástrofe y requieren acción inmediata; además, para estos autores, la información proporcionada por los MC está condicionada por los límites de espacio y tiempo de la profesión, así como por la capacidad limitada de la audiencia de medios para comprender problemas científicos 
complejos. Quesada, Blanco y Teruel (2015) destacan que son abundantes los estudios que llaman la atención sobre la ausencia de una política informativa coherente, en el sentido de que las noticias sobre ciencia no se cubren con ocasión de alertas o hallazgos científicos de relevancia, sino cuando resultan de la concatenación con eventos de naturaleza política, añadiendo a ello que la ideología de los MC constituye un elemento decisivo en el discurso editorial. Los resultados de su estudio confirman mayoritariamente que sus argumentos suelen prescindir de las voces científicas.

Castro, Reyes y Larrosa (2012), en su análisis del tratamiento de la prensa mexicana sobre temas ambientales, llegaron a dos conclusiones destacables: (1) el mayor peso concedido a la vertiente económica que a la degradación del medio, lo que coartaría las iniciativas proambientales de los lectores; y (2) la pobreza de sus formatos narrativos que oculta la verdadera complejidad de los problemas ambientales y, como consecuencia, ello promueve un papel pasivo del lector, impidiéndole la sensación de corresponsabilidad en la naturaleza de dichos problemas. Cumba (2020) abunda en este sentido cuando afirma que "todo hecho noticioso que esté relacionado a un conflicto ambiental con alguna de las corporaciones que el medio de comunicación mantiene tratos comerciales, se desvanece dentro de la agenda pública del medio para no afectar sus intereses económicos" (p. 130).

Portilla y González (2015) abordaron un análisis longitudinal de la prensa regional navarra sobre temas ambientales. Como resultados principales detectan un aumento de dicho tipo de noticias vinculado a acontecimientos internacionales, con el objetivo primordial de informar, aunque también aparecen otras con el de informar y concienciar, así como el de denunciar, lo que a juicio de las autoras restaría oportunidades a la ecoalfabetización. Por otro lado, también detectan una preocupante disminución de las noticias procedentes de fuentes institucionales, incluyendo las de índole especializada, pero un aumento de las de tinte más social, lo que va en detrimento de la información y sensibilización ambientales.

Francescutti, Tucho e Íñigo (2013) estudiaron las noticias ambientales durante un año en las cadenas de TV españolas, hallando que dicha información comparte con las demás noticias televisivas los rasgos de dramatismo, conflictividad, rareza, proximidad, culto a las celebrities y espectacularidad, diferenciándose de aquellas por su vertiente educativa y preventiva, y el protagonismo de las organizaciones de la sociedad civil. En concreto, los contenidos privilegiaron el daño ecológico, el debate sobre el CC y las acciones preventivas y de remediación, dejando poco espacio a la ciencia ambiental.

Si nos detenemos en analizar algunas de las causas del déficit e inadecuación de la información ambiental, al menos en el caso de España, debemos fijarnos en la formación que reciben a nivel universitario los futuros periodistas, formación ciertamente residual (Parratt, 2014), por lo que hay que suponer que la que adquieren los periodistas es de naturaleza autodidacta y asociativa. Así es en 1994 cuando se crea la Asociación de Periodistas de Información Ambiental (APIA) ${ }^{2}$.

\footnotetext{
2 http://www.apiaweb.org/
} 


\section{¿Satisface los requerimientos para cumplir con una labor alfabetizadora de la ciudadanía?}

Bonnett y Williams (1998) afirman que la comunicación ambiental ha asumido su papel formativo valiéndose de su poder de persuasión e intentando fomentar un espíritu significativo y autónomo. Bell (1994) propone una alianza entre periodistas y científicos para mejorar la información sobre el CC. Por otro lado, Lee (2008), basándose en investigaciones previas, sugiere que los $\mathrm{MC}$ tienen un papel fundamental en informar al público sobre el medio ambiente, pero pueden no estar realizando esta función correctamente.

\section{¿Podríamos integrar los MC en los currículos oficiales de la educación básica?}

Chu et al. (2007) opinan que, frente a un currículo integrado de la EA en Primaria, el uso de materiales de enseñanza como los MC (periódicos, revistas y programas de TV) puede ser efectivo para los niños pequeños. Klosterman, Sadler y Brown (2012), tras una revisión de la bibliografía al efecto, afirman que los profesores utilizan los MC en sus aulas para abordar cuestiones de sostenibilidad y son capaces de participar en prácticas de alfabetización mediática. Además, las intervenciones de los investigadores han demostrado que los estudiantes de ciencias de secundaria y los profesores tienen la capacidad de acceder, analizar, evaluar y crear medios.

\section{¿Cómo llevarlo a cabo?}

Conectando con el párrafo anterior, Klosterman, Sadler y Brown (2012) llevaron a cabo un estudio naturalístico con estudiantes y profesores de secundaria para investigar: (1) los usos que hacen de los MC para abordar los problemas de sostenibilidad a juicio de dichos profesores; (2) cómo los utilizan en sus aulas. Según sus resultados, los profesores y estudiantes participaron en el análisis y evaluación de los medios; sin embargo, el rango de estas prácticas fue algo limitado y variaba bastante entre profesores. Evaluaron la relevancia de los medios para sus clases y, en menor grado, suficiencia, credibilidad y usabilidad. Con todo, era menos probable que los maestros crearan oportunidades para que los estudiantes participaran en la evaluación. Este resultado, para los autores, revela las necesidades específicas para la formación inicial del profesorado y el desarrollo profesional en relación con la forma en que se deben utilizar los medios y los tipos de oportunidades que los alumnos deberían tener en las aulas. La creación de medios, la forma más alta y más exigente cognitivamente de alfabetización mediática, no se observó en este estudio, por lo que los autores se preguntan ¿por qué no poner el poder y el potencial de la producción de medios en sus manos?

Respondiendo a la recomendación de los anteriores autores, Marques y Reis (2017) estudiaron los efectos de la realización y difusión de vídeos digitales sobre el tema de la contaminación ambiental en las percepciones que los alumnos tienen sobre su propia capacitación para la acción y el desarrollo de competencias de activismo. Su realización y posterior divulgación también fueron utilizados como estrategia de activismo destinada a la educación. Los resultados mostraron una evolución positiva en la percepción de los estudiantes sobre su capacidad de acción, así como el desarrollo de competencias básicas para la acción colectiva basada en la investigación. 


\section{Reflexiones finales}

La revisión bibliográfica realizada nos permite disponer de una primera radiografía de la situación actual de la investigación sobre la utilización de los MC como herramientas de la EA.

En primer lugar, creemos necesario delimitar claramente lo que los MC aportan a la EA. Si partimos de que esta comporta información y, por tanto, puede contribuir a una alfabetización ambiental, también requiere concienciación y capacitación para la acción (incluyendo la vertiente actitudinal y, dentro de ella, la comportamental). Por consiguiente, se hace preciso considerar estas dimensiones para poder afirmar que los MC están contribuyendo globalmente a la EA.

Comenzaremos por la dimensión informativa. Parece incuestionable que los MC constituyen la principal fuente de información ambiental que nutre a los ciudadanos, aunque ello no significa que contribuya necesariamente a su formación, sino que presenta evidentes claroscuros.

Vamos a tratar de caracterizar la primera dimensión a través de la revisión bibliográfica realizada:

Como aspectos positivos parecen hallarse los siguientes:

- Contribuye a la información ambiental de la ciudadanía y a su alfabetización.

- Incrementa la concienciación ambiental, actitud, interés, comportamiento y compromiso político.

- Favorece la socialización de los temas ambientales.

- Ofrece protagonismo a las organizaciones de la sociedad civil.

- Genera un hueco en los MC como información con identidad propia.

No obstante, hay que remarcar la diferente incidencia de los $\mathrm{MC}$ según su naturaleza (p. ej., prensa/TV), sin que parezca existir un patrón único. A pesar de ello, la TV dispone del mayor potencial comunicativo en temas ambientales.

Como aspectos negativos encontramos:

- El peso de la información ambiental, aunque creciente, es mínimo frente al resto de la información.

- La información que proporcionan no conduce a fomentar una conciencia conservacionista.

- Los efectos positivos sobre la actitud suelen decaer en un tiempo relativamente breve.

- Están mediatizados por ideologías políticas e intereses económicos.

- Dependen excesivamente de eventos internacionales.

- Prima el sensacionalismo.

- Se presenta ligada a catástrofes.

- Busca maximizar la audiencia.

- Es descontextualizada.

- Muestra preferencia por contextos lejanos.

- No presenta la globalidad y complejidad de los problemas, así como explicaciones científicas para ellos. 
- No ofrece alternativas o soluciones para aquellos.

- Pueden contener errores que refuercen las concepciones sociales al respecto.

- Existe en ocasiones un divorcio entre la información periodística y la científica.

- Hay una escasa formación inicial en el periodismo ambiental como especialidad.Pueden contener errores que pueden reforzar.

En cuanto a la dimensión formativa, debemos considerar primero el papel de los propios lectores/espectadores. Se destaca las limitaciones cognitivas para comprender conceptos básicos relacionados con los problemas ambientales, pero también su pasividad ante ellos y la falta de espíritu crítico. Por otro lado, existen dudas razonables entre los educadores ambientales sobre las limitaciones de estos medios frente a la potencialidad del contacto con el medio natural.

Como sugerencias, se apuntan, entre otras, las siguientes:

- La comunicación ambiental puede aprovechar el poder de persuasión de los medios para educar.

- Sería conveniente una alianza entre científicos y periodistas para mejorar dicha comunicación.

- Aunque algunos profesores de ciencias utilizan los MC para explorar problemas socio-científicos y de sostenibilidad, su uso en educación para la sostenibilidad y alfabetización mediática es limitado, llegando a lo sumo a analizar los medios pero no a evaluarlos. En cuanto a la creación de estos medios por parte de los estudiantes es claramente infrecuente.

\section{Propuestas didácticas}

A la vista del análisis anterior creemos estar en disposición de proponer unas pautas de uso de los MC para la educación formal, con lo que trataríamos de dar respuesta a la pregunta no 4 que planteamos al inicio de este trabajo.

Dado el indiscutible peso que hoy día poseen los MC en la información que recibe el ciudadano y la dificultad de intervenir en aquellos para transformarlos en medios eficientes en el tratamiento de tal información, la alternativa que se torna más viable es la de integrarlos críticamente en el currículo de la educación formal a través de unas pautas dirigidas al profesorado y, por ende, a su alumnado. No obstante, somos conscientes de la limitada formación que dicho profesorado ha recibido, en general, sobre temáticas ambientales y también sobre el uso de los MC.

La ambientalización del currículo conllevaría entonces aprovechar los contenidos de distintas asignaturas para proponer actividades con los MC. Así se podrían trabajar algunos de los siguientes: consumismo, contaminación, residuos, deforestación, biodiversidad, agricultura/ganadería/pesca, agua, energía, turismo, organizaciones ecologistas. Ello permitiría trabajar diversas materias como Ciencias Sociales, Ciencias de la Naturaleza, Matemáticas, Lengua, Tecnología, ...

Para ello, un primer paso por parte del profesorado sería la selección del medio y de la tipología donde elegir la información relacionada con el contenido en sí. Si optamos, por ejemplo, por la TV y por la prensa, dispondríamos de las alternativas de la tabla 3. 
Tabla 3. Tipología de información ambiental en TV y prensa.

\begin{tabular}{ll}
\multicolumn{1}{c}{ TV } & \multicolumn{1}{c}{ Prensa } \\
Noticias & Noticias \\
Documentales & Reportajes \\
Programas infantiles & Opinión \\
Publicidad & Publicidad \\
& Turismo
\end{tabular}

Ante la pregunta de qué hacer con la información seleccionada, caben cuatro niveles de acción (tabla 4).

Tabla 4. Códigos para el análisis de la información ambiental. Fuente: adaptado de Klosterman, Sadler y Brown (2012, p. 59)

\section{Código}

Acceso Acceder a fuentes de medios no educativos e identificar la contribución educativa de esas fuentes para la ciencia.

Analizar Determinar el autor de los medios, la audiencia prevista, el mensaje previsto de la fuente o el contenido científico incrustado.

Evaluar Juzgar el valor de la fuente en términos de la precisión del contenido científico, su credibilidad, su adecuación, su relevancia o su usabilidad en términos de mejorar la alfabetización científica de uno.

Crear Creación de representaciones escritas y visuales de la ciencia para una audiencia pública geográficamente diversa.

En una primera fase (acceso), los estudiantes, con la asistencia del profesor, deberían poseer unas nociones básicas para buscar y contrastar información en medios de cierta solvencia (revistas, periódicos, páginas institucionales, centro de investigación...).

En la segunda (analizar) y la tercera (evaluar) pueden utilizarse como categorías de un modo conjunto una síntesis de algunas de las ventajas e inconvenientes señalados en la vertiente informativa anteriormente indicada. Nuestra propuesta sería la presente en la tabla 5. En la figura 2 mostramos la plantilla que hemos utilizado con estudiantes de grado y posgrado.

Tabla 5. Propuesta de categorías de análisis para la información ambiental de los MC.

\section{Categoría}

\section{Descripción}

\begin{tabular}{|c|c|}
\hline Actitud & Promueve (o no) la concienciación y capacitación para la acción a favor del medio. \\
\hline Organizaciones & Da protagonismo (o no) a las organizaciones ambientalistas. \\
\hline Medio & $\begin{array}{l}\text { Posee (o no) una línea con una orientación ideológica definida y/o vinculada a } \\
\text { intereses económicos evidentes. }\end{array}$ \\
\hline Mensaje & $\begin{array}{l}\text { Catastrofista / esperanzador: presenta un panorama negativo con tintes } \\
\text { sensacionalistas / presenta iniciativas o soluciones a los problemas ambientales. }\end{array}$ \\
\hline Complejidad & $\begin{array}{l}\text { Se presenta la información de un modo descriptivo / interpretativo / como opinión. } \\
\text { Se hace de un modo simplificado / complejo. Se muestran las causas y las } \\
\text { consecuencias. }\end{array}$ \\
\hline Precisión & Incluye (o no) errores desde el punto de vista científico. \\
\hline Contexto & $\begin{array}{l}\text { Próximo / lejano; se muestran (o no) los actores que intervienen (sociales y } \\
\text { ambientales). }\end{array}$ \\
\hline Evolución & $\begin{array}{l}\text { Se trata de un suceso sin antecedentes y consecuentes / o de un proceso en el que se } \\
\text { muestra la evolución en el tiempo. }\end{array}$ \\
\hline
\end{tabular}


Tipo de medio: prensa escrita, prensa digital, programa tv, pelicula, video en la red.

Formato: prensa escrita (noticia, reportaje, publicidad..), programa tv (documental, publicidad, infantil, noticias...), internet (pelicula, video youtube, página web...) Referencia: prensa escrita (título, nombre del medio, fecha y página), prensa digital (título, dirección URL), programa tv (título, cadena, fecha y hora de emisión), película (nombre y director), video (dirección URL).

Tratamiento de la información: mensaje (esperanzador/catastrofista); tratamiento (descriptivo/interpretativo; contexto lejano/próximo; como suceso/proceso) chenar las "6 W"s".

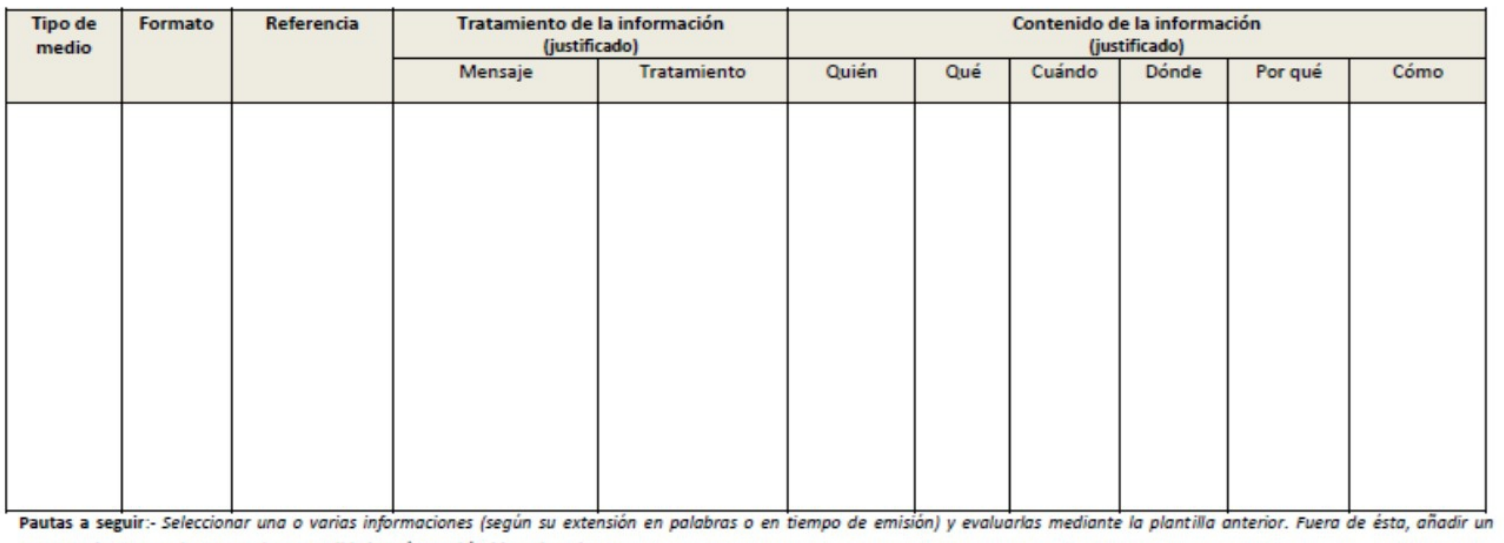

comentario que ayude a entender su posible interés en el ámbito educativo.

Figura 2. Ejemplo de plantilla de evaluación utilizada con estudiantes de universitarios de grado y posgrado.

Quedaría como reto por parte de los estudiantes el de la creación ad hoc de información ambiental que se ajuste a las categorías deseables descritas en la tabla 5, lo que se podría hacer en forma de texto escrito, reescribiendo una noticia, o en formato audiovisual y difundiéndola a través de plataformas o redes sociales.

\section{Referencias bibliográficas}

Bámaca-López, E. (2016). Comunicación del cambio climático en Guatemala. São Carlos: Pedro \& João Editores.

Barney, E. C., Mintzes, J., y Yen, C. F. (2005). Assessing Knowledge, Attitudes, and Behavior Toward Charismatic Megafauna: The Case of Dolphins. Journal of Environmental Education, 36(2), 41-55.

Bayma, V. V. (2013). El escepticismo de la comunicación incómoda. Las herramientas claves en la búsqueda del éxito en la comunicación medioambiental:un análisis de las piezas de comunicación de Al Gore y Bjorn Lomborg. Estudios sobre el Mensaje Periodístico, 19(1),

311-328. http://dx.doi.org/10.5209/rev_ESMP.2013.v19.n1.42524.

Bell, A. (1994). Media (mis)communication on the science of climate change. Public Understanding of Science, 3, 259-275.

Blum, A. (1987). Students' Knowledge and Beliefs concerning Environmental Issues in Four Countries. Journal of Environmental Education, 18(3), 7-13. https://doi.org/10.1080/00958964.1987.9942734.

Bonnett, M., y Williams, J. (1998). Environmental education and primary children's attitudes towards nature and the environment. Cambridge Journal of Education, 28(2), 159-174. 
Brothers, C. C., Fortner, R. W., y Mayer, V. J. (1991). The Impact of Television News on Public Environmental Knowledge. Journal of EnvironmentalEducation, 22(4), 22-29. https://doi.org/10.1080/00958964.1991.9943058.

Castro, E., Reyes, J., y Larrosa, J. (2012). El medio ambiente en la prensa nacional: marginalidad y escasa sustancia, en: J. M. Ramos, y E. Molina (Coords.), Comunicación ambiental en México: enfoques, experiencias, perspectivas (pp. 5177). Puebla (México): Montiel \& Soriano Editores S. A. de C. V.

Cumba, E. A. (2020). La educación ambiental en los medios televisivos. Estudio de caso: Oromar TV. Alteridad, 15(1), 125-138. https://doi.org/10.17163/alt.v15n1.2020.10.

Chu, H. E., Lee, E. A., Ko, H. R., Shin, D. H., Lee, M. N., Min, B. M., y Kang, K. H. (2007). Korean Year 3 Children's Environmental Literacy: A prerequisite for a Korean environmental education curriculum. International Journal of Science Education, 29(6), 731-746. https://doi.org/10.1080/09500690600823532.

Dotson, D. M., Jacobson, S. K., Kaid, L. L., y Carlton, J. S. (2012). Media Coverage of Climate Change in Chile: A Content Analysis of Conservative and Liberal Newspapers. Environmental Communication, 6(1), 64-81. https://doi.org/10.1080/17524032.2011.642078.

Engels, C. A., y Jacobson, S. K. (2007). Evaluating Long-Term Effects of the Golden Lion Tamarin Environmental Education Program in Brazil. Journal of Environmental Education, 38(3), 3-14.

Fortner, R.W. y Lyon, A.E. (1985). Effects of a Cousteau Television Special on Viewer Knowledge and Attitudes. Journal of EnvironmentalEducation, 16(3), 12-20.

Francescutti, L. P. (2010). La información científica en los telediarios españoles. Cuadernos de la Fundación Dr. Antonio Esteve, 21. https://www.esteve.org/libros/telediarios/.

Francescutti, L. P., Tucho, F., e Íñigo, A. I. (2013). El medio ambiente en la televisión española: análisis de un año de informativos. Estudios sobre el Mensaje Periodístico, $19(2)$, 683-701. http://dx.doi.org/10.5209/rev_ESMP.2013.v19.n2.43492.

Jiménez-Liso, M. R.; Perales, F. J., y Lara-Vargas, I. (2019). La mina de Aznalcóllar en Twitter años después del vertido: ¿controversia socioambiental espontánea o inducida? Estudios sobre el Mensaje Periodístico, 25(3), 1485-1498. https://doi.org/10.5209/esmp.66999.

Klosterman, M. L., Sadler, T. D., y Brown, J. (2012). Science Teachers' Use of Mass Media to Address Socio-Scientific and Sustainability Issues. Research in Science Education, 42, 51-74. https://doi.org/10.1007/s11165-011-9256-z.

Lee, E. B. (2008). Environmental Attitudes and Information Sources Among African American College Students. Journal of Environmental Education, 40(1), 29-42.

Lee, K. (2011). The role of media exposure, social exposure and biospheric value orientation in the environmental attitude-intention-behavior model in adolescents. Journal of Environmental Psychology, 31, 301-308. 
León, T., y Ruiz, J. D. (2012). Impacto mediático de los fenómenos medioambientales: la sequía en el sur de España durante el último medio siglo y su cobertura en prensa. Estudios sobre el Mensaje Periodístico, 18(2), 769-790. http://dx.doi.org/10.5209/rev_ESMP.2012.v18.n2.41045.

León, B. (ed.) (2014). Comunicar el cambio climático: de la agenda global a la representación mediática. Actas del XXVIII CICOM Congreso Internacional de Comunicación. Salamanca: Comunicación Social.

Lowe, T., Brown, T., Dessai, S., de França, M., Haynes, K., y Vincent, K. (2006). Does tomorrow ever come? Disaster narrative and public perceptions of climate change. Public Understanding of Science, 15, 435-457.

Marques, A. R., y Reis, P. (2017). Producción y difusión de vídeos digitales sobrecontaminación ambiental. Estudio de caso: Activismo colectivo basado en la investigación. Revista Eureka sobre Enseñanza y Divulgación de las Ciencias, 14(1), 215-226. http://hdl.handle.net/10498/18857.

Montero, J. M. (2008). Información ambiental en televisión. En: El periodismo ambiental. Análisis de un cambio cultural en España. Barcelona: Fundación Gas Natural.

Ojeda, F., Gutiérrez-Pérez, J. y Perales, F. J. (2011). TIC y Sostenibilidad: obstáculos y posibilidades para los educadores ambientales. Profesorado. Revista de currículum y formación del profesorado, 15(1), 263-313.

Ojeda, F., Perales, F. J., y Gutiérrez-Pérez, J. (2012). Evaluación de la calidad de webs y blogs sobre educación ecológica. Cultura y Educación, 24(1), 77-93.

Osorno, V. (2013). Las redes sociales como herramienta para la educación ambiental. Revista de Tecnología, 12(1), 55-65.

Östman, R. E., y Parker, J. L. (1987a). Impact of Education, Age, Newspapers, and Television on Environmental Knowledge, Concerns, and Behaviors. Journal of Environmental Education, 19(1), 3-9. https://doi.org/10.1080/00958964.1987.10801954.

Östman, R. E. y Parker, J. L. (1987b). A Public's Environmental Information Sources and Evaluations of Mass Media. Journal of Environmental Education, 18(2), 917. https://doi.org/10.1080/00958964.1987.9943483.

Östman, J. (2014). The Influence of Media Use on Environmental Engagement: A Political Socialization. Environmental Communication, 8(1), 92-109.

Paraskeva-Hadjichambi, D., Goldman, D., Hadjichambis, A. Ch., Parra, G., Lapin, K., Knippels, M. C., y Van Dam, F. (2020). Educating for Environmental Citizenship in Non-formal Frameworks for Secondary Level Youth. En: A.-Ch. Hadjichambis, P. Reis, D. Paraskeva-Hadjichambi, J. Cincera, J., Boeve-de Pauw, N. Gericke, M. C. Knippels (Eds.), Conceptualizing Environmental Citizenship for 21stCentury Education (pp. 213-235). Basilea: Springer Nature Switzerland A. G.

Parratt Fernández, S. (2014). El periodismo ambiental como especialidad en las universidades españolas. Prisma Social, 12, 335-363.

Perales, F. J. y García, N. (1999). Una propuesta de trabajo en el aula Educación Ambiental y medios de comunicación. Comunicar, 12, 149-155. 
Perales, F. J. (2010). Retos y dificultades para una educación ambiental informal. Alambique Didáctica de las Ciencias Experimentales, 64, 23-35.

Pérez-Nieto, T., y Perales, F. J. (2018). El Cambio Climático visto desde Internet: una evaluación de su contenido y mensaje. Estudios sobre el Mensaje Periodístico, 24(2), 1507-1524. https://doi.org/10.5209/ESMP.62231.

Portilla, I. y González, H. (2015). Noticias medioambientales en prensa regional: estudio de la evolución de temáticas, enfoques y fuentes. Estudios sobre el Mensaje Periodístico, 21(2), 1189-1206. http://dx.doi.org/10.5209/rev_ESMP.2015.v21.n2.50910.

Quesada, M., Blanco, E., y Teruel, L. (2015). El cambio climático en la prensa europea: discurso editorial en El País, Le Monde, The Guardian y Frankfurter Allgemeine Zeitung. Estudios sobre el Mensaje Periodístico, 21(1), 523-539. http://dx.doi.org/10.5209/rev_ESMP.2015.v21.n1.49109.

Quintanar, I. (2013). La educación ambiental formal en el contexto social de la educación informal y de los medios de comunicación. DELOS Desarrollo Local Sostenible, 6(18), 1-10.

Ramos, J. M., Molina, E., Hernández, H. G., y Sánchez, J. (2011). Prensa regional y medio ambiente:cobertura informativa en cuatro diarios de Puebla. Revista Iberoamericana de Comunicación, 21, 41-60.

Román-Núñez, Y. C., y Cuesta Moreno, O. J. (2016). Comunicación y conservación ambiental: avances y retos en Hispanoamérica. Revista Latina de Comunicación Social, 71, 15-39. http://dx.doi.org/10.4185/RLCS-2016-1082.

Said, A. M., Ahmadun, F.-R., Paim, L. H., y Masud, J. (2003). Environmental concerns, knowledge and practices gapamong Malaysian teachers. International Journal of Sustainability in Higher Education, 4(4), 305-313. http://dx.doi.org/10.1108/14676370310497534.

Sancho, J., Vilches, A., y Gil, D. (2010). Los documentales científicos como instrumentos de educación para la sostenibilidad. Revista Eureka para la Enseñanza y Divulgación Científica, 7(3), 667-681.

Segerberg, A., y Bennett, W. L. (2011). Social Media and the Organization of Collective Action: Using Twitter to Explore the Ecologies of Two Climate Change Protests. The Communication Review, 14(3), 197-215. http://dx.doi.org/10.1080/10714421.2011.597250.

Takahashi, B., Tandoc, E. C., Duan, R., y Van Witsen, A. (2017). Revisiting Environmental Citizenship: The Role of Information Capital and Media Use. Environment and Behavior, 49(2) 111-135.

Taddicken, M. (2013). Climate change from the user's perspective: The impact of mass media and Internet use and individual and moderating variables on knowledge and attitudes. Journal of Media Psychology: Theories, Methods, and Applications, 25(1), 39-52. https://doi.org/10.1027/1864-1105/a000080

Teksoz, G., Sahin, E., y Tekkaya-Oztekin, C. (2012). Modeling Environmental Literacy of University Students. Journal of Science and Education Technology, 21(1), 157166. http://dx.doi.org/10.1007/s10956-011-9294-3 
Teksoz, G. T., Boone, J. W., Tuzun, O. Y., y Tekkaya-Oztekin, C. (2014). An evaluation of the environmental literacy of preservice teachers in Turkey through Rasch analysis. Environmental Education Research, 20(2), 202-227. http://dx.doi.org/10.1007/s10956-011-9294310.1080/13504622.2013.768604.

Thompson-Saud, G., Gelcich, S. y Barraza, J. (2018). Marine environmental issues in the mass media: Insights from television, newspaper and Internet searches in Chile. Oceanand Coastal Management, 165, 154-160. https://doi.org/10.1016/j.ocecoaman.2018.08.015.

Unión Internacional de Conservación de la Naturaleza (UICN)(1970). Carta de Nevada.

Wakefield, S. E. L., y Elliott, S. J. (2003). Constructing the News: The Role of Local Newspapers in Environmental Risk Communication. The Professional Geographer, 55(2), 216-226.

Weingart, P., Engels, A., y Pansegrau, P. (2000). Risks of communication: discourses on climate change in science, politics, and the mass media. Public Understanding Science, 9, 261-283. 